\title{
AVALIAÇÃO DA UTILIZAÇÃO DE PRENSA DE ROLOS EM CIRCUITOS DE COMINUIÇÃO DE UM MINÉRIO DE FERRO ITABIRITICO*
}

\author{
Kelly Cristina Ferreira ${ }^{1}$ \\ Maria Auxiliadora Mendes Aguiar ${ }^{2}$ \\ Ângelo Quintiliano Nunes da Silva ${ }^{3}$
}

\begin{abstract}
Resumo
Nas usinas de beneficiamento mineral os gastos com cominuição podem chegar a $80 \%$ do total. Diante disso, as indústrias minerais vêm buscando alternativas para a diminuição do consumo energético, dentre as quais destaca-se a utilização de prensas de rolos (HPGR). No presente estudo foram avaliados alguns parâmetros utilizados no dimensionamento de prensas de rolos, tais como capacidade específica, pressão de moagem e energia necessária. Além disso foi avaliado o impacto do aumento da umidade na produtividade do equipamento e determinada a curva de distribuição granulométrica dos produtos e abrasividade do minério. Resultados mostraram que valores de umidade superiores a $5 \%$ causam grande prejuízo à capacidade específica do equipamento. Devido à alta abrasividade observada nos ensaios (27 a $48 \mathrm{~g} / \mathrm{t}$ ), percebe-se haver necessidade de estudos mais aprofundados para que se possa aplicar de maneira eficiente a prensa de rolos no material estudado.
\end{abstract}

Palavras-chave: Minério de ferro itabiritico; Cominuição; Prensa de rolos; HPGR.

\section{EVALUATION OF THE USE OF HIGH PRESSURE GRINDING ROLLS FOR ITABIRITIC IRON ORE COMMINUTION CIRCUIT}

\section{Abstract}

In mineral processing plants comminution expenses may reach $80 \%$ of the total. Therefore, the mineral industries are seeking alternatives to reduce energy consumption, standing out the use of high pressure grinding rollers (HPGR). In the present study it was evaluated some parameters used in the design of HPGR, such as specific capacity, grinding pressure and energy required. The study also aimed to assesses the impact of moisture variation on ore feed in respect to specific grinding capacity and product size distribution. It was also analyzed the abrasiveness of the ore. Results demonstrated that moisture values greater than $5 \%$ cause great damage to the specific capacity of the equipment. It was observed high abrasiveness in the trials (27 to $48 \mathrm{~g} / \mathrm{t}$ ). Further studies are needed to implement efficiently the roller press in the studied material.

Keywords: Iron ore; Comminution; High pressure grinding rollers; HPGR.

1 Engenheira Metalurgista, Mestranda em Tecnologia Mineral, Escola de Engenharia, Universidade Federal de Minas Gerais, Belo Horizonte, Brasil.

2 Engenheira Química, Mestre em Tecnologia Mineral, Doutoranda, Escola de Engenharia, Universidade Federal de Minas Gerais, Belo Horizonte, Brasil.

3 Engenheiro de Minas, Mestre em Tecnologia Mineral, Doutorando, Escola de Engenharia, Universidade Federal de Minas Gerais, Belo Horizonte, Brasil. 


\section{INTRODUÇÃO}

Este trabalho apresenta um estudo sobre a busca de alternativas para o circuito de cominuição de um minério de ferro itabirítico.

A tecnologia de prensa de rolos (HPGR) ilustrada na Figura 1 não é inteiramente nova. O conceito pode ter sido concebido com base em prensas de rolos que foram largamente utilizadas na briquetagem de materiais de britagem relativamente fácil [1]. Porém, a moderna conceituação foi introduzida por volta de 1984 pelo Prof. Dr. Engenheiro Alemão Klaus Schonert, nascido em 1927 e falecido em 2011 aos 84 anos de idade. A prensa de rolos tem funcionamento relativamente simples e, após as modificações, vem sendo aplicada com sucesso a materiais mais resistentes e abrasivos. De acordo com Ribeiro et al. [2] atualmente, em minério de ferro, são utilizadas para britagem grosseira, moagem de plebbes de moinhos autógenos, remoagem pré-pelotização e briquetagem.

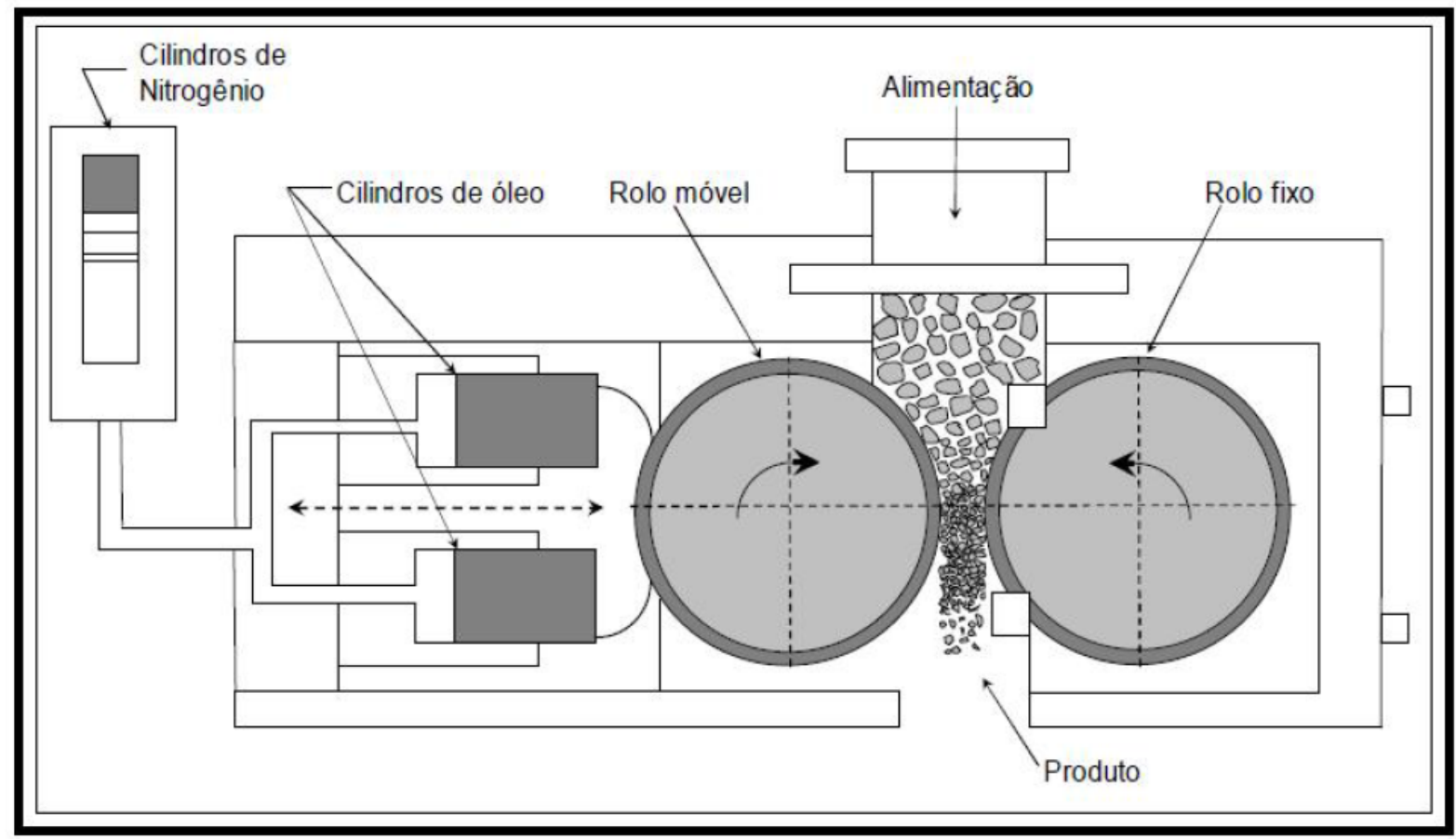

Figura 1: Prensa de rolos (Valery e Yankvoic apud [1])

Segundo Schonert [3] os equipamentos de cominuição, tais como britadores, moinhos e prensas reduzem os materiais pelos mecanismos de tensão de compressão ou por força de tração.

Nas prensas de rolos um leito ou um conjunto de partículas é comprimido através da lenta aplicação de carga sobre as partículas, fazendo com que ocorra o colapso estrutural dos grãos, de forma que o material é reduzido com maior eficiência energética do que, por exemplo, em moinhos e britadores convencionais. Daí a grande motivação para estudos de utilização deste equipamento nos circuitos de cominuição.

Aplicações da prensa de rolos em muitas alternativas de circuitos tem resultado em economia de energia de $10-50 \%$ em comparação com operações de circuitos fechados de moinho de bolas [4]. Além disso, devido às microtrincas criadas por forças de compressão altas em um leito de partículas, o índice de trabalho de Bond é reduzido [5]. 
Os principais objetivos do presente estudo foram a avaliação de parâmetros utilizados no dimensionamento de prensas de rolos, tais como capacidade específica, pressão de moagem e energia necessária, avaliação do impacto do aumento da umidade na produtividade do equipamento e determinação da distribuição granulométrica dos produtos e da abrasividade do minério.

\section{MATERIAIS E MÉTODOS}

Para a realização do estudo, um total de $1400 \mathrm{~kg}$ de ROM de minério de ferro itabirítico foi preparado e enviado ao centro de pesquisas da Krupp / Polysius na Alemanha.

A preparação da amostra consistiu na obtenção de material britado a $-31,5 \mathrm{~mm}$. A densidade encontrada para a amostra foi de $1,984 \mathrm{t} / \mathrm{m}^{3}$. A análise mineralógica demonstrou composição de $63,4 \%$ de quartzo $\left(\mathrm{SiO}_{2}\right)$ e $36,6 \%$ de hematita $\left(\mathrm{Fe}_{2} \mathrm{O}_{3}\right)$. A análise granulométrica da amostra estudada encontra-se na Figura 2 abaixo.

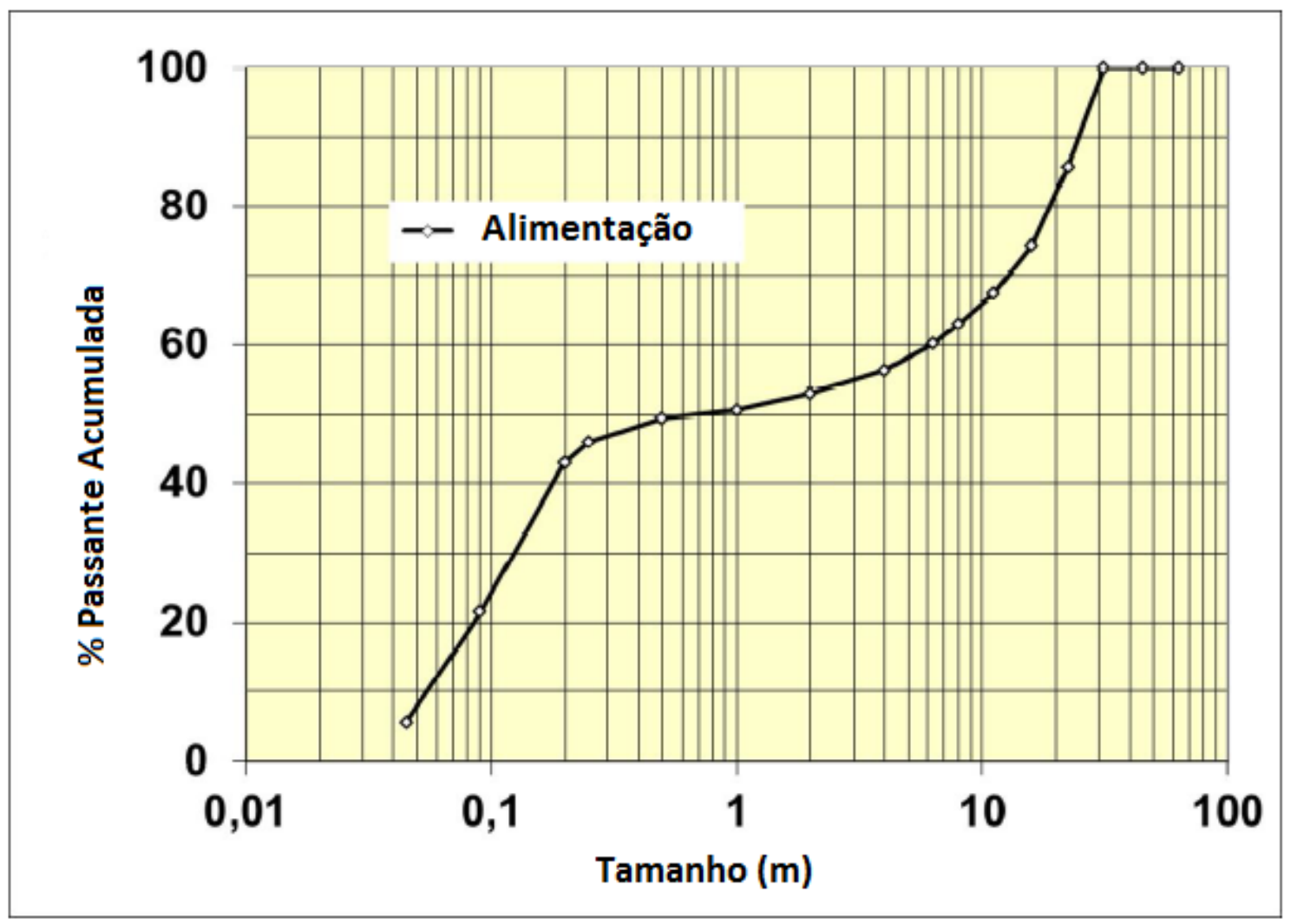

Figura 2: Análise granulométrica da alimentação do teste

Foram realizados 5 testes com uma massa de $225 \mathrm{~kg}$ cada. Estas foram preparadas com diferentes umidades, conforme demonstrado em tabela abaixo.

Tabela 1: Amostras utilizadas nos testes de prensa de rolos

\begin{tabular}{cc}
\hline Amostra & Umidade (\%) \\
\hline M1 & 5,0 \\
M2 & 5,0 \\
M3 & 5,0 \\
M4 & 3,0 \\
M5 & 8,0 \\
\hline
\end{tabular}


Os testes foram realizados na unidade piloto MAGRO, uma prensa de rolos semiindustrial da Polysius. O diâmetro dos rolos é de 0,95 m e largura de 0,35 m, com velocidade de rotação de 0,20 a $1,0 \mathrm{~m} / \mathrm{s}$.

Os rolos são compostos de múltiplos segmentos, revestidos com pinos de carbeto de tungstênio. Um dos rolos é fixo enquanto o outro móvel. Na Figura 3 encontra-se uma fotografia da unidade piloto na qual foram realizados os experimentos.

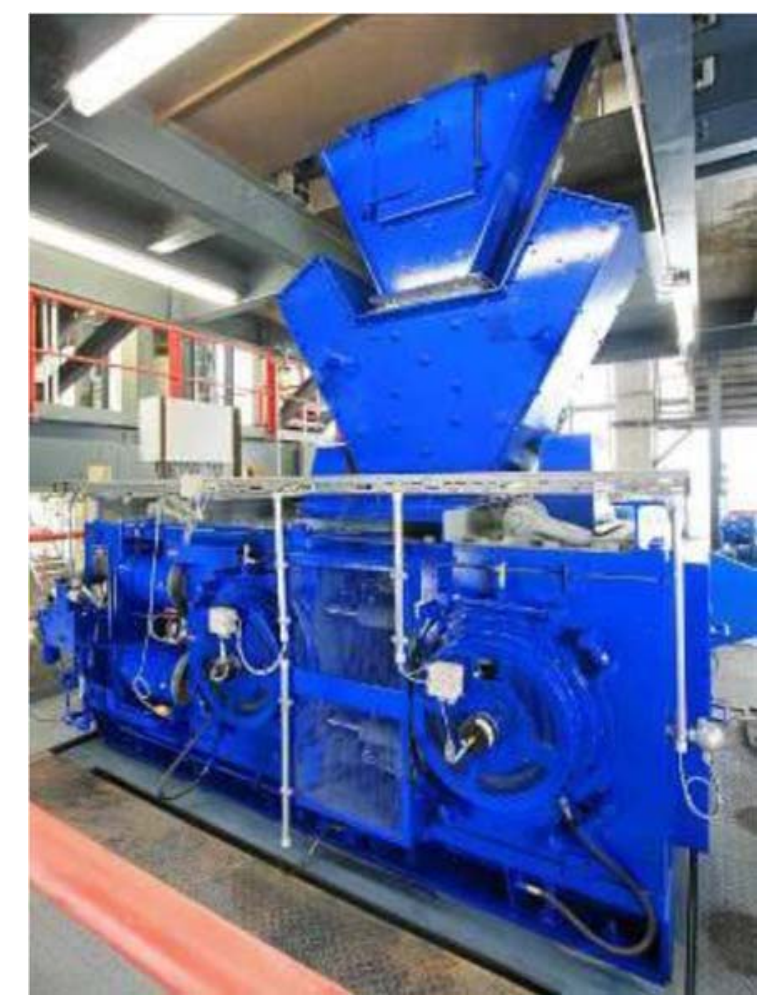

Figura 3: Unidade piloto da prensa de rolos (HPGR)

Durante os testes, a velocidade de rotação dos rolos e a força de compressão foram mantidas constantes.

Além dos testes em prensa piloto, foi realizado teste para determinação do índice de abrasividade do material, com o objetivo de determinar a taxa de desgaste do revestimento. Para este teste, a amostra foi britada abaixo de $3,15 \mathrm{~mm}$. Os testes foram conduzidos com força de compressão específica de $4 \mathrm{~N} / \mathrm{mm}^{2}$ e a umidade foi alterada entre 0 e $1 \%$.

\section{RESULTADOS E DISCUSSÃO}

\subsection{Avaliação da Influência da Umidade na Capacidade Específica}

A influência do teor de umidade na capacidade específica é mostrada abaixo para uma força de compressão específica de $3,7 \mathrm{~N} / \mathrm{mm}^{2}$. 


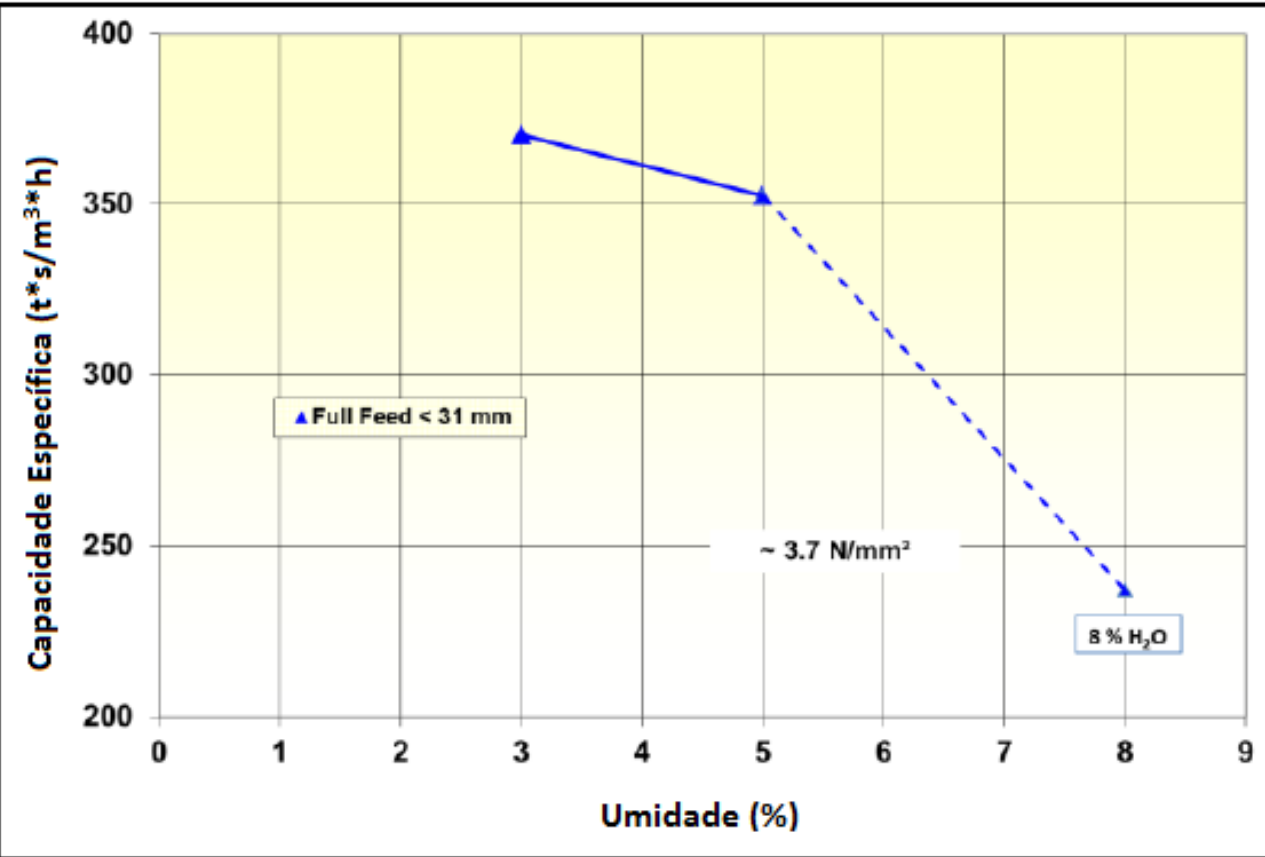

Figura 4. Variação da capacidade específica em função do teor de umidade da alimentação

O teor de umidade até $5 \%$ tem um menor impacto na capacidade específica que, nessa faixa, variou de 380 a $340 \mathrm{t}^{*} \mathrm{~s} /\left(\mathrm{m}^{3 *} \mathrm{~h}\right)$.

\subsection{Avaliação da Potência Absorvida, Pressão de Moagem e Consumo Energético}

Maiores pressões de moagem resultam em maior potência absorvida pelo material. A potência absorvida é, tipicamente, uma função linear da pressão de moagem. No gráfico abaixo, verifica-se esta correlação.

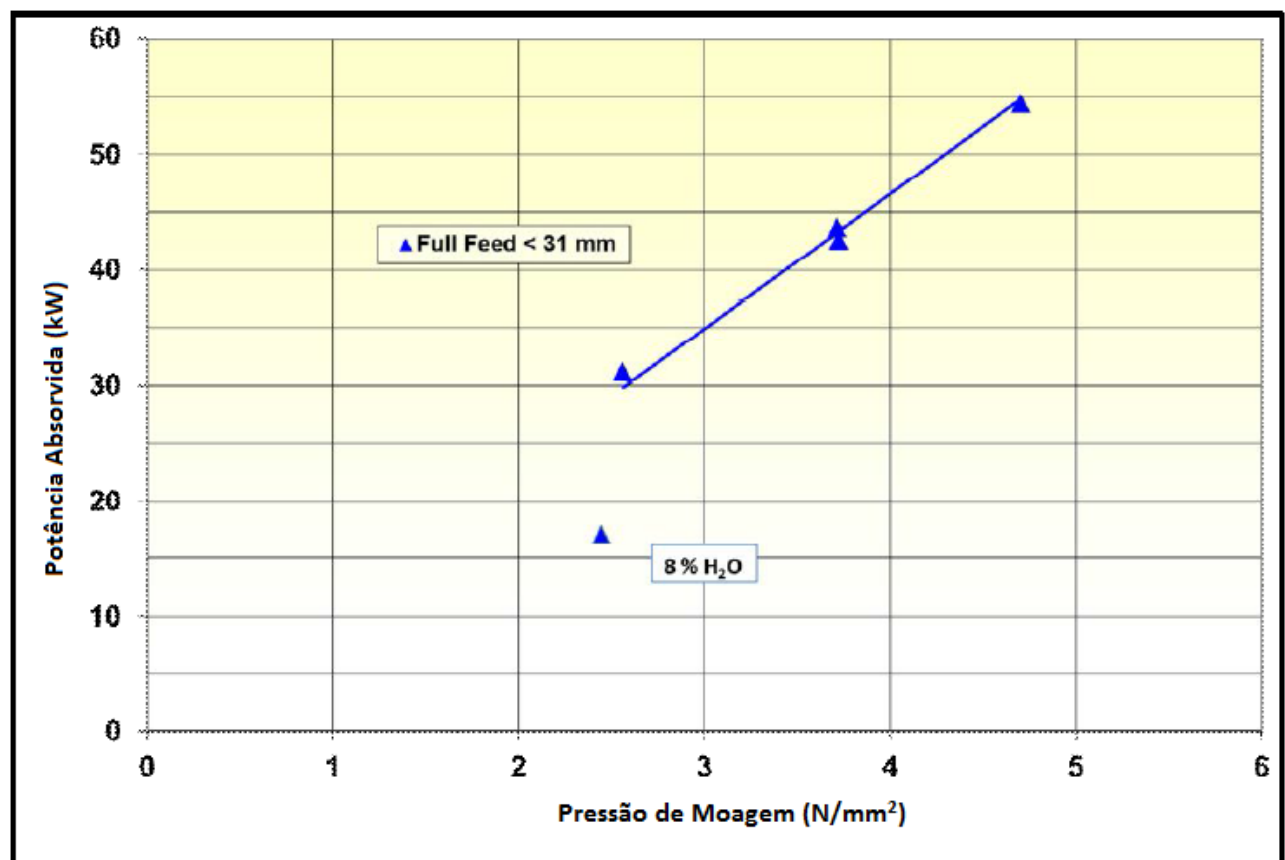

Figura 5. Correlação entre a pressão de moagem e a potência absorvida 
O consumo especifico de energia é consequência da potência absorvida e da capacidade atingível.

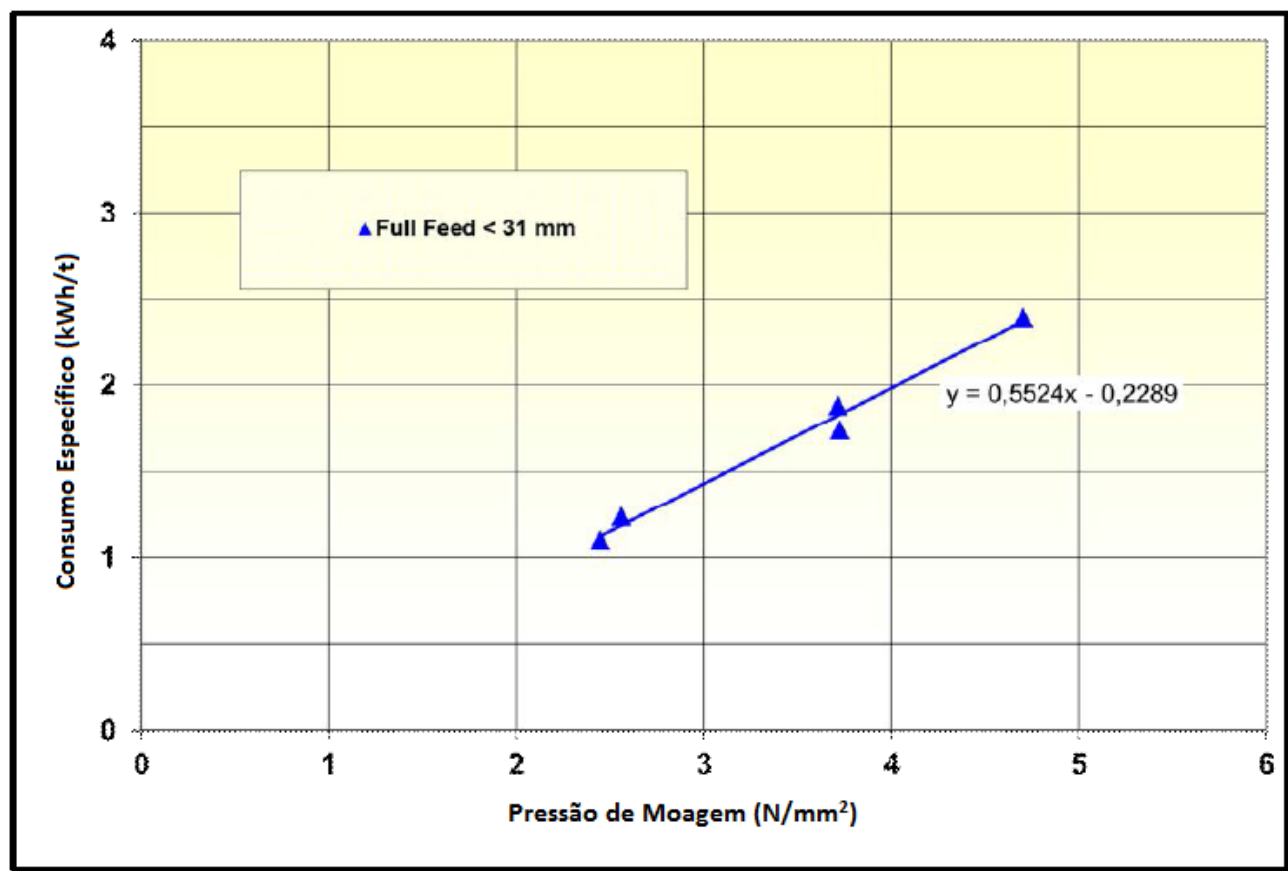

Figura 6. Correlação entre a pressão de moagem e o consumo específico

O consumo específico de energia alcançados ficaram entre 1,1 e 2,39 kWh/t no intervalo de pressões testadas. O consumo especifico em $3,5 \mathrm{~N} / \mathrm{mm}^{2}$ ficou em $1,7 \mathrm{kWh} / \mathrm{t}$.

\subsection{Avaliação da Distribuição Granulométrica do Produto e a Pressão de Moagem}

A distribuição granulométrica do produto da HPGR é controlada, dentro de determinados limites, pela aplicação da pressão de moagem. A amostra da descarga teve as porções laterais e central coletadas separadamente e suas frações foram analisadas individualmente. A correlação entre o produto gerado e a pressão de moagem da amostra global (descarga central e descarga lateral) é mostrada na Figura 5. O produto gerado é representado pela porcentagem passante acumulada em $200 \mu \mathrm{m}, 1 \mathrm{~mm}$ e $6,3 \mathrm{~mm}$. 


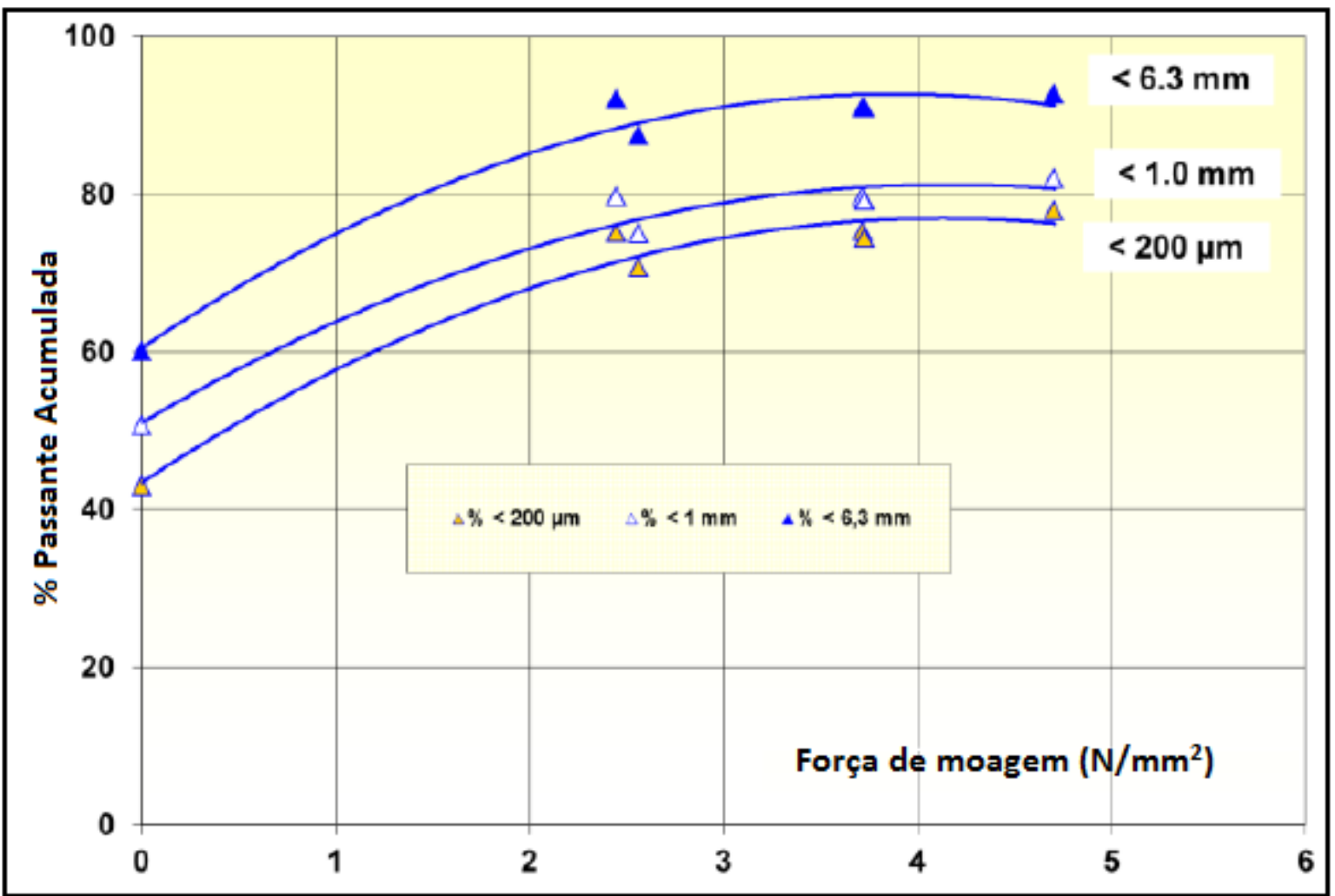

Figura 7. Distribuição granulométrica do produto para diferentes valores de pressão de moagem

Um aumento da \% passante acumulada para maiores forças de moagem é encontrada.

Forças de moagem acima de $2,5-4 \mathrm{~N} / \mathrm{mm}^{2}$ resultaram em aumento da \%passante pequena.

\subsection{Avaliação da Taxa de Desgaste}

Para a determinação do índice de abrasividade, foram realizados dois testes. Os testes foram conduzidos em amostras britadas abaixo de $3,15 \mathrm{~mm}$. A pressão de moagem utilizada foi de $4 \mathrm{~N} / \mathrm{mm}^{2}$ e a umidade variou de 0 a $1 \%$. Os resultados do teste são apresentados na tabela abaixo.

\begin{tabular}{|ccccc|}
\hline & $\begin{array}{c}\text { Top Size da } \\
\text { Alimentação } \\
(\mathrm{mm})\end{array}$ & $\begin{array}{c}\text { Umidade } \\
(\% \mathrm{H} 2 \mathrm{O})\end{array}$ & $\begin{array}{c}\text { Pressão de } \\
\text { Moagem } \\
(\mathrm{N} / \mathrm{mm} 2)\end{array}$ & $\begin{array}{c}\text { Taxa de } \\
\text { Desgaste } \\
(\mathrm{g} / \mathrm{t})\end{array}$ \\
\hline A1 & 3,15 & 0 & 4 & 27,91 \\
A2 & 3,15 & 1 & 4 & 48,47 \\
\hline
\end{tabular}


Tabela 2: Resultados dos ensaios para índices de abrasividade do material

\begin{tabular}{|c|c|c|c|c|}
\hline & $\begin{array}{l}\text { Top Size da } \\
\text { Alimentação } \\
(\mathrm{mm})\end{array}$ & $\begin{array}{l}\text { Umidade } \\
\left(\% \mathrm{H}_{2} \mathrm{O}\right)\end{array}$ & $\begin{array}{l}\text { Pressão de } \\
\text { Moagem } \\
\left(\mathrm{N} / \mathrm{mm}^{2}\right)\end{array}$ & $\begin{array}{c}\text { Taxa de Desgaste } \\
(\mathrm{g} / \mathrm{t})\end{array}$ \\
\hline $\mathrm{A} 1$ & 3,15 & 0,0 & 4 & 27,91 \\
\hline $\mathrm{A} 2$ & 3,15 & 1,0 & 4 & 48,47 \\
\hline
\end{tabular}

Os resultados mostrados na Tabela 2 indicam que o material tem uma alta abrasividade. A taxa de desgaste, em escala industrial, é calculada levando em conta o diâmetro e a velocidade dos rolos, o tipo e o comprimento dos pinos (studs) aplicados e as características do material. Com estes resultados, foi estimado para este material uma vida útil de 5200 horas, o que para este projeto em questão, foi considerado baixo. Maiores investigações deverão ser realizadas para a validação da utilização da prensa no circuito de cominuição.

\section{CONCLUSÃO}

Neste estudo, a utilização de prensa de rolos em um circuito de cominuição de minério de ferro itabiritico foi avaliada.

Os testes demonstraram a grande influência que um teor de umidade maior que $8 \%$ causou na capacidade especifica, diminuindo-a drasticamente. Além disso, os resultados constaram que as pressões de moagem acima de $2,5-4 \mathrm{~N} / \mathrm{mm}^{2}$ resultaram em aumento da porcentagem passante pequena.

Por fim, os resultados dos ensaios de índice de abrasividade indicaram que o material possui grande abrasividade, sendo estimado então, uma vida útil de 5200 horas do revestimento, o que expõe a necessidade de um estudo mais aprofundado sobre a utilização da prensa para esta aplicação.

\section{Agradecimentos}

Os autores agradecem a ABM pela oportunidade de divulgação deste trabalho, e ao CNPQ e à CAPES pelo apoio ao PPGEM.

\section{REFERÊNCIAS}

1 Alves, V.K.;Metodologia para simulação e escalonamento de prensas de rolos; Tese de doutorado, Universidade Federal de Minas Gerais; 2012; 140p.;

2 Ribeiro, F.S.; Russo, J.F.C.; Costa, T.; Aplicação de prensas de rolos em minério de ferro; REM: R. Esc. Minas; 2010; 399-404;

3 Schonert, $\mathrm{K}$, ,.A first survey of grinding with high-compression roller mills, International Journal of Mineral Processing, 22:401-412.

4 Patzelt, N., Knecht, H., Baum, W., The metallurgical potential of high pressure roll grinding, XX International Mineral Processing Congress. GDMB, Aachen, Germany, 1997, p.155-164.

5 Aydogan, N.A.; Ergun, L.;Benzer, H.; High pressure grinding rolls (HPGR) application in the cement industry. Minerals Engineering, 2005, p.130-139. 